\title{
Análisis tridimensional del comportamiento dinámico de una fortificación ante un evento sísmico inducido
}

\author{
Tridimensional analysis of the dynamic behaviour of a fortification under an induced seismic \\ event \\ Fecha de entrega: 12 de diciembre 2016 \\ Fecha de aceptación: 18 de abril 2017
}

\section{David Troncoso ${ }^{1}$ y Marcelo Bacco ${ }^{2}$}

\author{
${ }^{1}$ Departamento de Obras Civiles, Universidad Técnica Federico Santa María, Av. España 1680, Valparaíso, Chile, \\ david.troncosoc@alumnos.usm.cl \\ ${ }^{2}$ Arcadis Chile, Antonio Varas 621, Providencia, Santiago, Chile, mbacco@arcadis.cl
}

Los estallidos de roca son un riesgo que ha ido en aumento en excavaciones alrededor de todo el mundo. La principal razón de esta tendencia es el aumento de la profundidad en la que se están realizando las correspondientes actividades. La capacidad que poseen los elementos de una cierta fortificación frente a este tipo de eventos ha sido exhaustivamente analizada durante los últimos veinticinco años. Principalmente en términos de su capacidad de absorber energía sísmica. Sin embargo, aún existe una gran incertidumbre en lo que respecta a la cantidad de energía que efectivamente transfiere un evento sísmico a la fortificación, es decir, la demanda de energía. El estudio que se presenta a continuación es un modelo tridimensional realizado en el programa Flac3D. Este modelo tiene como principal objetivo evaluar la energía ejercida por el macizo rocoso sobre la fortificación ante eventos sísmicos de distintas magnitudes y comparar estos resultados con los resultados de los ensayos publicados a la fecha.

Palabras clave: estallido de roca, demanda de energía, Flac3D, macizo rocoso, evento sísmico, energía sísmica
The rockburst risk has been increasing worldwide, as the tendency is to execute activities in deeper environments. In most projects affected by seismicity, seismic monitoring is operating on the rockmass, and, according to the dynamic behaviour registered at these devices, seismic intensity assessment is provided for each seismic event. The measure of support capacity to absorb energy when submitted to these events has been the subject of intensive research over the last twenty five years. However, the amount of energy effectively received by the support (demand of energy), still is uncertain under real events conditions. The present work corresponds to a tridimensional modelling executed in Flac3D software. The main objective of this study is to estimate the seismic energy transferred by the rockmass to the underground support considering different seismic magnitudes and to compare them with published results.

Keywords: rockburst, energy demand, Flac3D, rockmass, seismic event, seismic energy

\section{Introducción}

Cuando se producen procesos de rupturas y deformaciones dentro de las cavidades de un macizo rocoso, se puede producir la generación de ondas elásticas, las cuales pueden alcanzar los sectores donde se estén realizando las operaciones correspondientes al proyecto. A este fenómeno se le denomina evento sísmico inducido. Cuando las ondas radiadas por un evento sísmico alcanzan el sector de excavación se pueden generar daños. Si este daño se genera en forma violenta y repentina se le denomina estallido de roca. Sin embargo, se debe hacer notar que existen distintas definiciones propuestas respecto a qué se puede entender como estallido de roca o evento sísmico, pero, en general responden a una idea similar (Diederichs, 2014).

El primer estallido de roca registrado data de antes del año 1900 en la mina de carbón de Upper-Silesian, Polonia (Ortlepp, 2006). Y desde ese momento a la fecha se han producido eventos de distinta envergadura alrededor de todo el mundo. Sin duda, el país con mayores problemas de sismicidad inducida es Sudáfrica: posee 6 minas 
ubicadas a más de $3000 \mathrm{~m}$ de profundidad, las cuales tienen regularmente eventos de muy alta magnitud (Potvin et al., 2007) y en promedio registra aproximadamente 20 accidentes fatales anualmente de acuerdo a la base de datos Samrass (Potvin, 2009). Las mineras australianas, por su parte, han tenido problemas de actividad sísmica durante todo un siglo. Ya en 1917 se registraron accidentes fatales y lesionados en la localidad de Kalgoorlie (Kagoorlie Miner, 1917). Desde 1960 se ha reportado actividad sísmica en Broken Hill (Rauert y Tully, 1998) y Charlotte (Mikula y Poplawski, 1995). Entre 1996 y 1998 se han registrado al menos tres accidentes fatales (Potvin et al., 2000); y más recientemente, el altamente conocido caso de la minera Beaconsfield en Tasmania, que resultó en la muerte de un trabajador y otros dos trabajadores atrapados dentro de la mina (Mellick, 2007). En Chile, los primeros indicios se presentaron en la mina El Teniente, los cuales se pudieron identificar con certeza el año 1976. El primer evento mayor ocurrió en 1980 y obligó a la minera a detener sus actividades (Ortlepp y Stacey, 1994). El caso más actual y emblemático corresponde a un accidente fatal ocurrido el año 2014 en el marco de la realización del proyecto Nuevo Nivel Mina en la división El Teniente, el cual aún se encuentra en investigación (Pizarro, 2014), pero se cree podría deberse a un estallido de roca.

Debido a todos estos antecedentes, los estallidos de roca son un tema que ha sido sometido a intensa investigación, sobretodo en la década de 1990 cuando se comenzaron a realizar nuevos proyectos a mayor profundidad en distintos lugares del mundo. Sin embargo, tal como es reconocido por varios investigadores (Heal, 2010; Potvin et al., 2010; Brummer, 1999), el fenómeno de los estallidos de roca y los eventos sísmicos inducidos aún no es del todo comprendido. Es por esta razón que esta investigación pretende aportar mayor entendimiento del fenómeno desde el punto de vista de la modelación matemática, realizada por medio del programa comercial Flac3D, apuntando a responder una de las varías incertidumbres que existen en este campo de investigación: la transferencia de energía sísmica entre un macizo rocoso y una determinada fortificación.

\section{Disposiciones generales}

La geometría del modelo consiste en una intersección de túneles de medio punto que representan la excavación; una cavidad esférica ubicada en una determinada posición, que representa a la fuente sísmica; y los bordes del modelo representados por un paralelepípedo que rodea a todos los demás elementos. En la Figura 1 se muestran las dimensiones de todas estas componentes.
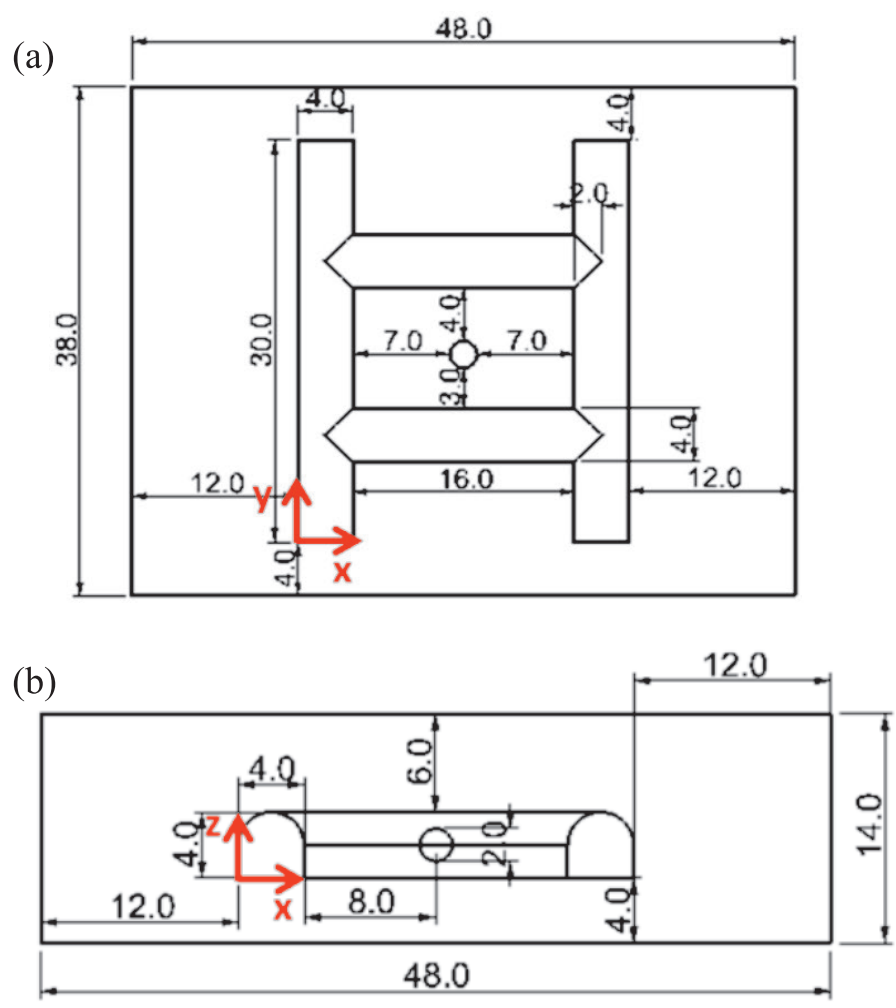

Figura 1: a) Planta y b) vista frontal de la excavación, foco y bordes del modelo realizado, dimensiones en $\mathrm{m}$

\section{Caracterización geotécnica del macizo rocoso}

\section{Consideraciones}

La caracterización geotécnica del macizo rocoso fue realizada en base a los valores de parámetros publicados por distintos autores a la fecha como se indica a continuación. No se representa una situación de un sitio en particular sino que se estiman los valores de parámetros de la roca intacta y el macizo rocoso de acuerdo a las condiciones en las cuales se pueden detonar los estallidos de roca.

\section{Propiedades del macizo rocoso antes de la falla}

Los parámetros de la roca intacta fueron supuestos considerando los resultados publicados por: Hoek y Brown (1980), Rahn (1986), Johnson y de Graff (1988), Goodman (1989), Walthan (1999), Wyllie (1999), Obert y Duvall (1967) y Farmer (1968). Se consideró el modelo 
de falla propuesto por Hoek y Brown (1980) y sus últimas actualizaciones (Hoek et al., 2002).

Tabla 1: Propiedades de la roca intacta

\begin{tabular}{|l|c|c|c|}
\hline Parámetro & Notación & Unidades & Valor \\
\hline Peso unitario & $\gamma$ & ton $/ \mathrm{m}^{3}$ & 2.7 \\
\hline $\begin{array}{l}\text { Resistencia a la compresión } \\
\text { uniaxial de la roca intacta }\end{array}$ & $\sigma_{\mathrm{ci}}$ & $\mathrm{MPa}$ & 150 \\
\hline Parámetro roca intacta & $m_{\mathrm{i}}$ & - & 9 \\
\hline $\begin{array}{l}\text { Módulo de deformación de la } \\
\text { roca intacta }\end{array}$ & $E_{\mathrm{i}}$ & $\mathrm{GPa}$ & 40 \\
\hline Coeficiente de Poisson & $v$ & - & 0.2 \\
\hline Parámetro de voladura de roca & $D$ & - & 0.8 \\
\hline
\end{tabular}

El escalamiento de propiedades del macizo rocoso a partir de las propiedades de la roca intacta se realizó mediante las expresiones propuestas por Hoek et al. (2002) y Hoek y Diederichs (2006).

$$
\begin{aligned}
& m_{\mathrm{b}}=m_{\mathrm{i}} e^{\frac{G S I-100}{28-14 D}} \\
& s=e^{\frac{G S I-100}{9-D}} \\
& a=\frac{1}{2}+\frac{1}{6}\left(e^{-\frac{G S I}{15}}-e^{-\frac{20}{3}}\right) \\
& E_{\mathrm{m}}=E_{\mathrm{i}}\left(0.02+\frac{1-\frac{D}{2}}{1+e^{\frac{60+15 D-G S I}{11}}}\right)
\end{aligned}
$$

El valor de GSI supuesto fue de 70. Este valor está basado en la calidad geotécnica mínima que poseen los macizos rocosos capaces de acumular energía y generar un evento sísmico (Diederichs, 2014). En la Tabla 2 se muestran los resultados obtenidos a partir de las expresiones (1), (2), (3) y (4).

Tabla 2: Propiedades escaladas del macizo rocoso

\begin{tabular}{|l|c|c|c|}
\hline Parámetro & Notación & Unidades & Valor \\
\hline Índice de Resistencia Geológica & GSI & - & 70 \\
\hline Módulo de elasticidad & $E_{\mathrm{m}}$ & $\mathrm{GPa}$ & 17.3 \\
\hline \multirow{2}{*}{$\begin{array}{l}\text { Parámetros del modelo de falla } \\
\text { Hoek-Brown }\end{array}$} & $a$ & - & 0.5 \\
\cline { 2 - 4 } & $m_{\mathrm{b}}$ & - & 1.694 \\
\cline { 2 - 4 } & $s$ & - & 0.0226 \\
\hline
\end{tabular}

\section{Propiedades del macizo rocoso después de la falla}

Para caracterizar los elementos plastificados se consideró una reducción de su calidad geotécnica. Este proceso se realizó considerando un valor residual de GSI a partir de la siguiente expresión propuesta por Cai et al. (2007).

$G S I_{\mathrm{r}}=G S I e^{-0.0134 G S I}$

\section{Metodología de modelación Fortificación}

Se supuso un caso desfavorable para la fortificación, es decir, un refuerzo solo diseñado para ser sometido a cargas estáticas o cargas dinámicas bajas. Para este efecto se utilizó el método de Barton et al. (1974), considerando un valor de $S R F=100$ y las condiciones geométricas mostradas en la Figura 1. Obteniéndose de esta forma la fortificación detallada en la Tabla 3 y Figura 2.

Tabla 3: Características de la fortificación considerada en el modelo

\begin{tabular}{|l|l|}
\hline Tipo de refuerzo & Características \\
\hline Shotcrete & $100 \mathrm{~mm}$ de espesor \\
\hline Pernos & $\begin{array}{l}\text { Radiales, } 3 \mathrm{~m} \text { de largo, } \\
25 \mathrm{~mm} \text { de diámetro, patrón } 1 \mathrm{~m} \mathrm{x} 1 \mathrm{~m}\end{array}$ \\
\hline
\end{tabular}

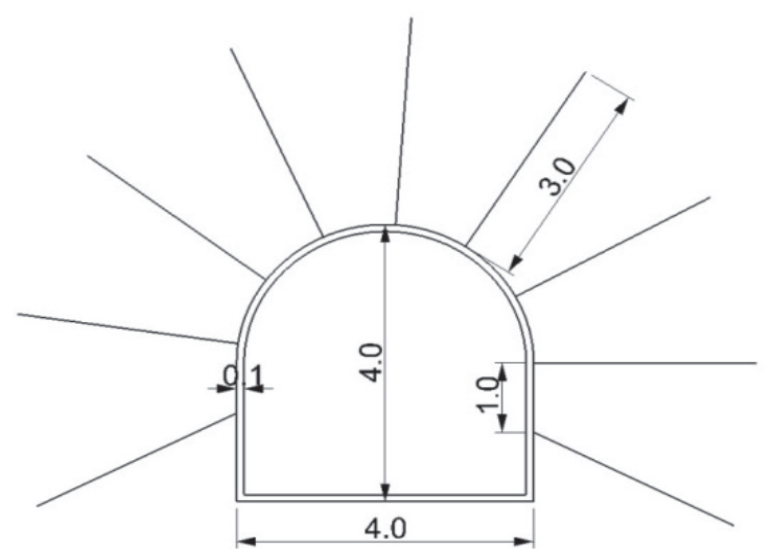

Figura 2: Fortificación considerada para el modelo, dimensiones en $\mathrm{m}$

\section{Esfuerzos in-situ}

El tensor de esfuerzos in situ, al igual que la caracterización geotécnica del macizo rocoso, no corresponde a una situación real de estudio sino que representa una situación desfavorable de esfuerzos tanto en magnitud como esfuerzo desviatorio. Los valores fueron estimados a partir de los esfuerzos reales medidos en distintos lugares del 
mundo (Hoek y Brown, 1980). El tensor de esfuerzos in situ considerado es el siguiente en MPa:

$$
\overline{\bar{\sigma}}=\left(\begin{array}{ccc}
-50 & 0 & 0 \\
0 & -29 & 0 \\
0 & 0 & -15
\end{array}\right)
$$

\section{Bordes del modelo}

Los bordes del modelo fueron considerados, en una etapa estática, como rotulados en los bordes paralelos a los planos verticales (XZ, YZ) y empotrados en el borde inferior paralelo al plano horizontal (XY). Este supuesto se muestra esquemáticamente en la Figura 3. En una etapa dinámica se consideran bordes absorbentes en todos los límites del modelo.

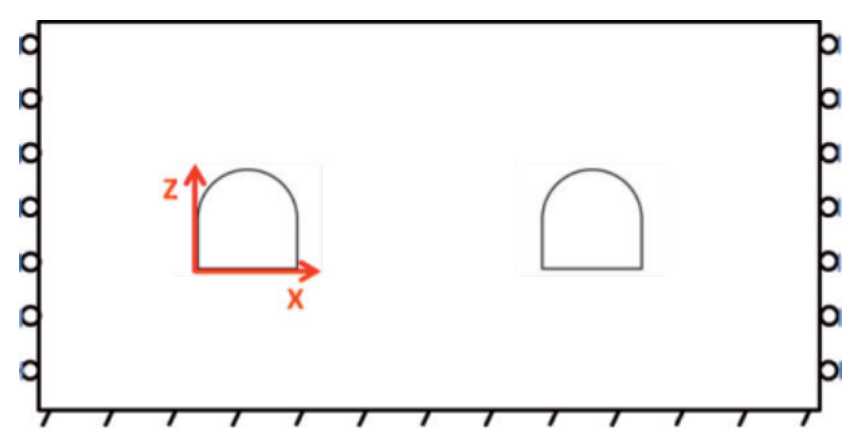

Figura 3: Esquema de los bordes del modelo en una etapa estática

\section{Amortiguamiento}

El amortiguamiento local de los elementos se modeló según la siguiente expresión de Rayleigh (Figura 4).

$$
\xi_{i}=\frac{1}{2}\left(\frac{\alpha}{\omega_{i}}+\beta \omega_{i}\right)
$$

Este comportamiento se obtuvo de forma iterativa y solo por razones de estabilidad numérica y tiempos de cálculo, ya que simplemente se supuso un valor muy bajo de este parámetro por tratarse de elementos de roca (Kramer, 1996).

\section{Input dinámico}

El input dinámico aplicado en la fuente sísmica fue representado mediante un registro de velocidades. Este registro de velocidades consiste en la superposición de pulsos sinusoidales con frecuencias entre $0.1 \mathrm{~Hz}$ hasta 300 Hz. El resultado, luego de normalizar esta superposición, se muestra en la Figura 5.

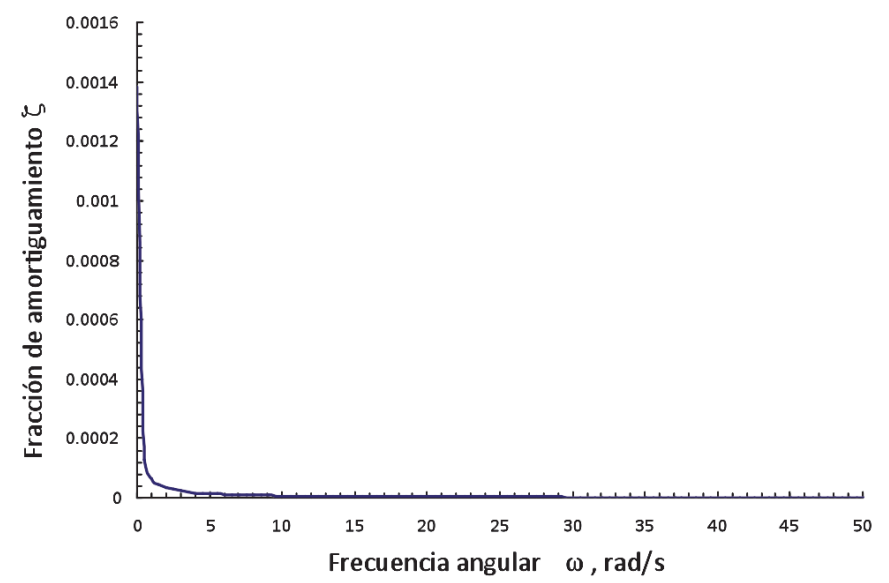

Figura 4: Curva de amortiguamiento local de Rayleigh

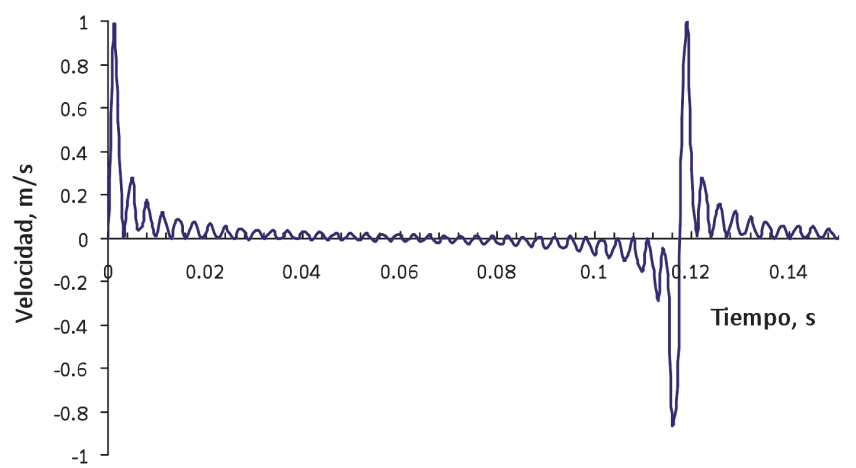

Figura 5: Registro de velocidades de amplitud unitaria aplicado en el foco

\section{Propiedades de la fortificación}

\section{Pernos (cables)}

Los pernos fueron idealizados como elementos elastoplásticos denominados cables, y la lechada como resortes que conectan el macizo rocoso y dichos elementos tipo cable. Las propiedades supuestas, basadas en parámetros recomendados por los desarrolladores del programa (Flac3D, 2013), tanto de los elementos cable y de la lechada idealizada se presentan en la Tabla 4.

\section{Shotcrete (shell)}

El comportamiento del shotcrete fue idealizado como un material lineal elástico sin límite de resistencia. En el modelo, el shotcrete fue representado por un conjunto de elementos denominados shell. Las propiedades supuestas para los elementos tipo shell se detallan en la Tabla 5 (Flac3D, 2013). 
Tabla 4: Propiedades de los pernos y la lechada

\begin{tabular}{|l|c|c|c|}
\hline Parámetro & Notación & Unidades & Valor \\
\hline $\begin{array}{l}\text { Módulo de deformación } \\
\text { del cable }\end{array}$ & $E$ & $\mathrm{GPa}$ & 200 \\
\hline $\begin{array}{l}\text { Fuerza de fluencia en el } \\
\text { cable, } \sigma_{\mathrm{y}}=420 \mathrm{MPa}\end{array}$ & $F_{\mathrm{y}}$ & $\mathrm{kN}$ & 206 \\
\hline $\begin{array}{l}\text { Área transversal del ca- } \\
\text { ble, diámetro } 25 \mathrm{~mm}\end{array}$ & $A$ & $\mathrm{~m}^{2}$ & $4.9 \cdot 10^{-4}$ \\
\hline $\begin{array}{l}\text { Rigidez de corte de la le- } \\
\text { chada por unidad de largo }\end{array}$ & $k_{\mathrm{g}}$ & $\mathrm{N} / \mathrm{m}^{2}$ & $1.7 \cdot 10^{9}$ \\
\hline $\begin{array}{l}\text { Resistencia de corte de } \\
\text { la lechada por unidad de } \\
\text { largo }\end{array}$ & $c_{\mathrm{g}}$ & $\mathrm{N} / \mathrm{m}^{2}$ & $1.7 \cdot 10^{9}$ \\
\hline
\end{tabular}

Tabla 5: Propiedades de los elementos tipo shell

\begin{tabular}{|l|c|c|c|}
\hline Parámetro & Notación & Unidades & Valor \\
\hline Módulo de deformación & $E$ & $\mathrm{GPa}$ & 15 \\
\hline Razón de Poisson & $v$ & - & 0.2 \\
\hline
\end{tabular}

\section{Resultados}

\section{Calibración de magnitud}

Para estimar la magnitud asociada a cada input de velocidades se utilizó la expresión propuesta por Potvin et al. (2010). Esta expresión relaciona el valor peak de la velocidad en un determinado punto (Peak Particle Velocity $\mathrm{PPV})$ con la distancia a la fuente sísmica $R$.

$$
\mathrm{PPV}=C \frac{10^{\frac{1}{2}\left(m_{L}+1.5\right)}}{R+R_{0}}
$$

Donde $C=0.2-0.3, R$ es la distancia al punto en cuestión y $R_{0}$ es el radio de la fuente sísmica. Se midió el máximo del valor absoluto del módulo de la velocidad en distintos puntos del modelo (Figura 6), sin realizar la excavación, y

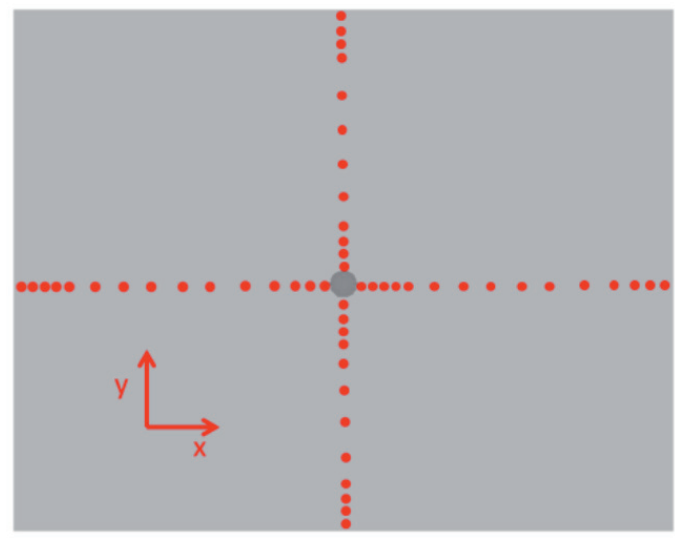

Figura 6: Puntos de calibración de magnitud considerando distintos valores de amplitud para el registro de velocidades aplicado (ver Figura 5).

De acuerdo a (8), la relación entre la PPV y el término 1/ $\left(R+R_{0}\right)$ debiese ser lineal. En la Figura 7 se muestran los resultados obtenidos al graficar los resultados de estas dos variables. En la Tabla 6 se muestran los resultados de las regresiones realizadas a estos datos considerando $R_{0}=1 \mathrm{~m}$ (ver Figura 1).

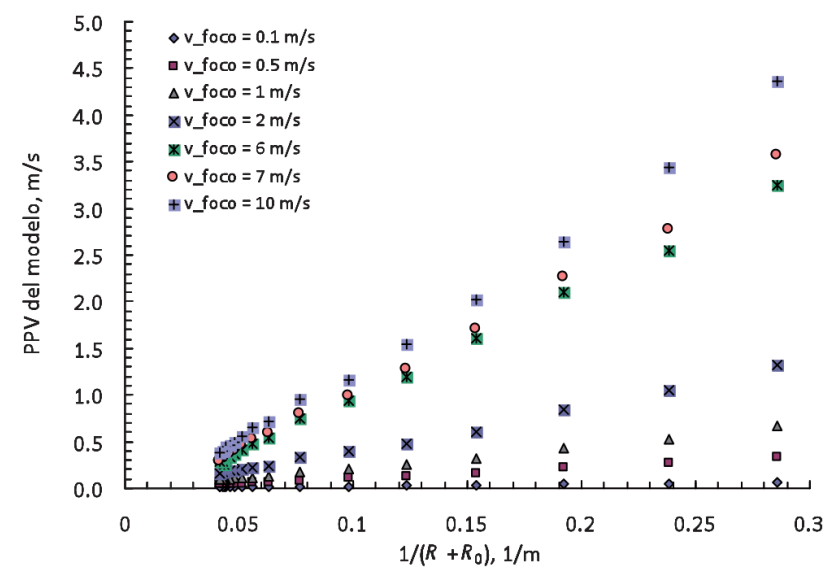

Figura 7: Valores de PPV medidos en el modelo versus $1 /\left(R+R_{0}\right)$

Tabla 6: Resultados de las regresiones obtenidas de los datos de PPV medidos

\begin{tabular}{|c|c|c|c|}
\hline $\mathrm{v}_{\text {foco }}, \mathrm{m} / \mathrm{s}$ & $r^{2}$ & $C$ & Magnitud local Richter $m_{\mathrm{L}}$ \\
\hline 10 & 0.997 & $0.2-0.3$ & $1.95 \mathrm{a} 2.3$ \\
\hline 7 & 0.998 & $0.2-0.3$ & $1.77 \mathrm{a} 2.12$ \\
\hline 6 & 0.999 & $0.2-0.3$ & $1.7 \mathrm{a} 2.05$ \\
\hline 2 & 0.994 & $0.2-0.3$ & $0.87 \mathrm{a} 1.22$ \\
\hline 1 & 0.996 & $0.2-0.3$ & $0.25 \mathrm{a} 0.61$ \\
\hline 0.5 & 0.996 & $0.2-0.3$ & $-0.33 \mathrm{a} 0.02$ \\
\hline 0.1 & 0.996 & $0.2-0.3$ & $-1.73 \mathrm{a}-1.37$ \\
\hline
\end{tabular}

\section{Amplificación de velocidades}

Se compararon los valores de PPV medidos sin ninguna excavación presente, obteniendo estos datos de los resultados discutidos en la sección anterior de calibración de magnitud, con los valores de PPV medidos considerando la presencia de la excavación y fortificación. Estos resultados se analizaron en el perfil que se muestra en la Figura 8.

Este proceso se hizo para distintas amplitudes del registro. La forma de los resultados obtenidos para todos los inputs aplicados se muestran en la Figura 9.

Si se define el factor de amplificación en la excavación 
FAE, como el cociente entre el valor de PPV medido en el borde de la excavación y el valor de PPV en el mismo punto antes de realizar la excavación, se tienen los siguientes resultados mostrados en la Tabla 7.
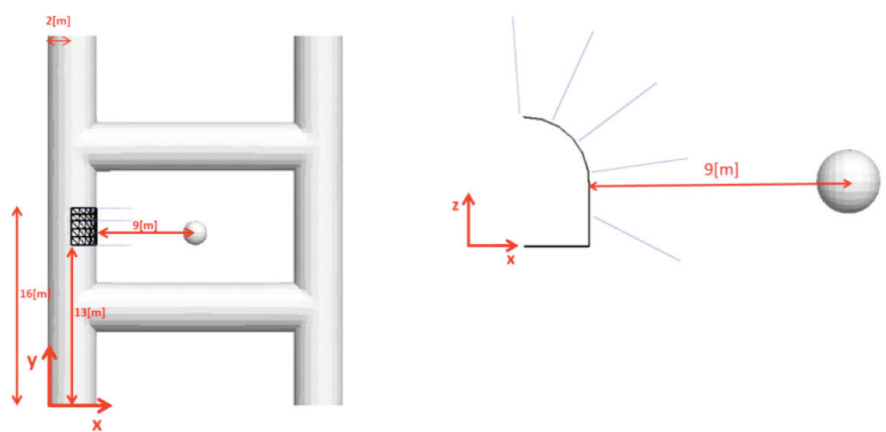

Figura 8: Perfil de medición de velocidades

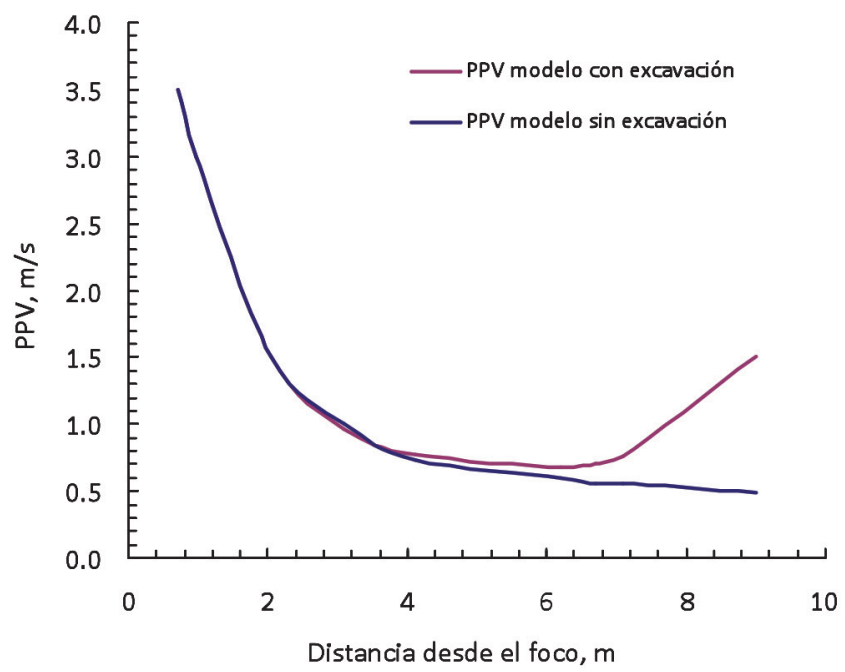

Figura 9: Gráfico general del perfil de velocidades obtenido

Tabla 7: Resultados del factor FAE obtenido para distintas magnitudes aplicadas en el foco

\begin{tabular}{|c|c|}
\hline$m_{\mathrm{L}}$ & FAE \\
\hline 1.7 a 2.05 & 2.8 \\
\hline 0.87 a 1.22 & 3.8 \\
\hline 0.25 a 0.61 & 4.2 \\
\hline-0.33 a 0.02 & 4.8 \\
\hline-1.73 a -1.37 & 5.2 \\
\hline
\end{tabular}

\section{Transferencia de energía}

La transferencia de energía desde el macizo hacia la fortificación se calculó de acuerdo a las fuerzas y los desplazamientos desarrollados en los elementos shell. Se define la densidad de energía recibida por un elemento shell como:
$E_{\mathrm{pS}}=\frac{\int_{r_{1}}^{r_{2}} \overrightarrow{F_{t S}} \cdot d \vec{r}}{A_{t}}$

donde $\overrightarrow{F_{t S}}$ es la fuerza tributaria aplicada sobre un punto de un elemento shell, $d \vec{r}$ es el vector desplazamiento del punto analizado y $A_{\mathrm{t}}$ es el área tributaria correspondiente al punto. Se midió la densidad de energía absorbida por los elementos shell para el sector de la fortificación que se muestra en la Figura 10.
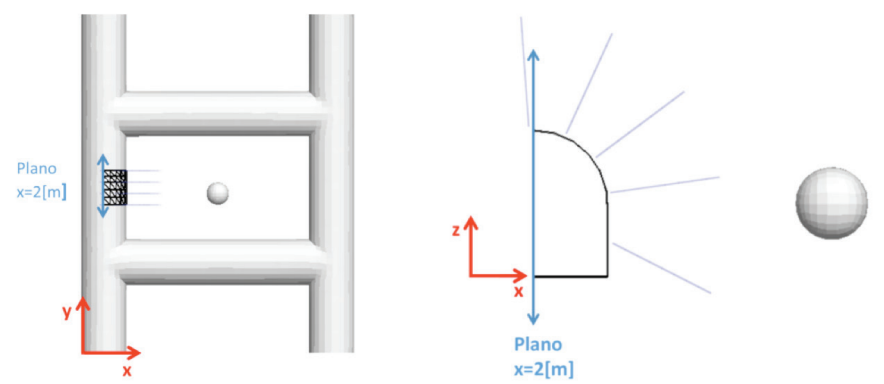

Figura 10: Plano considerado para el mapa de contorno de densidades de energía

Los resultados de las distintas densidades de energía se proyectaron sobre el plano $x=2$, como se muestra en la Figura 10. No obstante, en este caso se compararon los valores obtenidos con la recopilación de resultados publicados a la fecha (Potvin et al., 2010). A partir de este análisis, se obtiene la falla de un sector de la fortificación para eventos de magnitud $m_{\mathrm{L}}=0.25$ a 0.61 , como se muestra en la Figura 11.

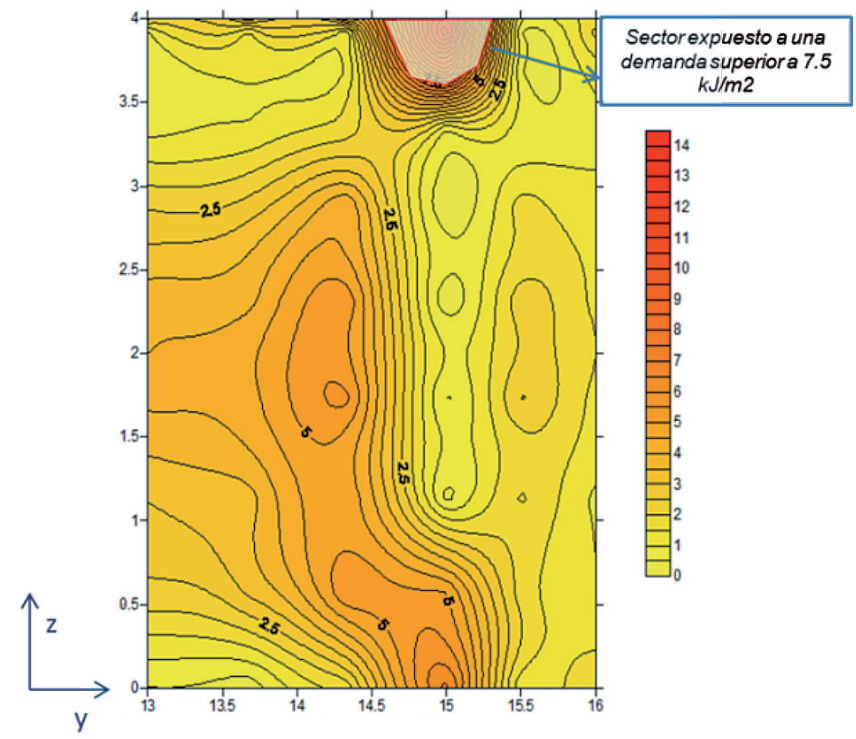

Figura 11: Mapa de contornos obtenido con un input asociado a un evento de magnitud $\mathrm{m}_{\mathrm{L}}=0.25$ a 0.61 , medido en $\mathrm{kJ} / \mathrm{m}^{2}$ 
En la Tabla 8 se muestran los valores máximos de la densidad de energía obtenida en el mapa de contornos de la Figura 11 para eventos en distintos rangos de magnitud. El resto de los valores del mapa de contornos está en la misma proporción que los valores máximos.

Tabla 8: Valores máximos obtenidos en los mapas de contorno asociados a distintos rangos de magnitud

\begin{tabular}{|c|c|}
\hline Magnitud $m_{\mathrm{L}}$ & $\begin{array}{c}\text { Demanda de energía máxima, } \\
\mathrm{kJ} / \mathrm{m}^{2}\end{array}$ \\
\hline 1.7 a 2.05 & 48.0 \\
\hline 0.87 a 1.22 & 25.6 \\
\hline 0.25 a 0.61 & 14.4 \\
\hline-0.33 a 0.02 & 7.1 \\
\hline-1.73 a -1.37 & 1.5 \\
\hline
\end{tabular}

\section{Discusiones y conclusiones}

Los resultados de la calibración de magnitudes son consistentes con la expresión de decaimiento de velocidades utilizada (Hoek y Brown, 1980), en términos de las correlaciones obtenidas. Este resultado debe ser entendido con detenimiento, ya que si bien las regresiones indican una clara tendencia de las velocidades a decaer ajustándose a la curva de la expresión (8), el amortiguamiento no fue incluido como un elemento de análisis, y sería válido preguntarse qué tan influyente pudo haber sido este parámetro si se hubiesen considerado valores más altos. De todas formas, el amortiguamiento en rocas es por lo general bajo (Kramer, 1996), particularmente bajo si se está considerando un macizo rocoso de alta calidad geotécnica y una distancia pequeña entre el foco sísmico y la excavación. Dado lo anteriormente mencionado, se puede concluir que los resultados obtenidos de modelar un foco sísmico mediante una cavidad esférica, en la cual se aplicó un registro de velocidades sintético con distintas amplitudes, son coherentes con la fórmula empírica utilizada para valores bajos de amortiguamiento.

En el modelo, los valores de energía que absorbieron los elementos tipo shell indican que un sismo de magnitud $m_{\mathrm{L}}$ $=0.25-0.61$, produce la falla de un sector considerable de la fortificación. De acuerdo a lo expuesto por Oterlepp (1992), esta magnitud no está asociada a los eventos más destructivos, por lo tanto se podría decir que dichos valores corresponden a un rango relativamente bajo de magnitudes. También se debe notar que el valor de $7.5 \mathrm{~kJ} / \mathrm{m}^{2}$ de capacidad se consideró suponiendo que el shotcrete poseía al menos fibras de refuerzo, ya que de otra forma tendría aún menos capacidad de absorción (Hoek y Brown, 1980). Esta capacidad se podría aumentar significativamente en caso de usar mallas de refuerzo tipo chain link que pueden alcanzar los $13 \mathrm{~kJ} / \mathrm{m}^{2}$, o los refuerzos con la metodología lacing que pueden alcanzar más de $30 \mathrm{~kJ} / \mathrm{m}^{2}$ (Hoek y Brown, 1980). Sin embargo, no se pueden extrapolar tan sencillamente los resultados de esta modelación ya que usar dicho tipo de refuerzos implicaría modificar las propiedades de los elementos de la fortificación en el modelo, y es de esperar, que los valores de energía transferidos varíen.

Se puede discutir acerca de la posición del foco elegida, ya que $9 \mathrm{~m}$ es una situación bastante desfavorable. Sin embargo, como se argumenta en Kaiser et al. (1996) y Diederichs (2014), los eventos sísmicos inducidos no son fenómenos aislados y pueden generar otros eventos en otros sectores del macizo rocoso, incluso de mayor intensidad que el original, de modo que en términos prácticos el foco sísmico puede estar ubicado a esta distancia o a cualquier otra. Debido a lo anteriormente mencionado, en base a los resultados del modelo y todo lo mencionado en el párrafo anterior, se puede concluir que no es recomendable usar este tipo de fortificación en sectores donde haya riesgo de estallidos de rocas de magnitudes sobre $\operatorname{los} m_{\mathrm{L}}=0.25$.

\section{Referencias}

Barton, N., Lien, R. and Lunde, J. (1974). Engineering classification of rock masses for the design of tunnel support. Rock Mechanics 6(4), 189-236

Brummer, R.K. (1999). Simple truths about rockbursts. Second South African Rock Engineering Symposium, SARES 99, ISRM Regional Symposium, Johannesburg

Cai, M., Kaiser, P.K., Tasaka, Y. and Minami, M. (2007). Determination of residual strength parameters of jointed rock masses using the GSI system. International Journal of Rock Mechanics \& Mining Sciences 44(2), 247-265

Diederichs, M.S. (2014). When does brittle failure become violent? Spalling and rockburst characterization for deep tunneling projects. World Tunnel Congress 2014. Iguassu Falls, Brazil, 1-10 
Farmer, I.W. (1968). Engineering properties of rocks. E \& FN Spon

Flac3D (2013). Fast Lagrangian Analysis of Continua in 3 Dimensions. Dynamic Analysis. Itasca Consulting Group

Goodman, R.E. (1989). Introduction to rock mechanics. John Wiley \& Sons

Heal, D. (2010). Observations and analysis of incidences of rockburst damage in underground mines. $\mathrm{PhD}$ thesis, The University of Western Australia, Perth, Australia

Hoek, E. and Diederichs, M.S. (2006). Empirical estimation of rock mass modulus. International Journal of Rock Mechanics and Mining Sciences 43(2), 203-215

Hoek, E. and Brown, E.T. (1980). Underground excavation in rock. The Institution of Mining and Metallurgy, London

Hoek, E., Carranza-Torres, C. and Corkum, B. (2002). HoekBrown criterion - 2002 edition. 5th North American Rock Mechanics Symposium and the 17th Tunnelling Association of Canada Conference NARMS-TAC, Toronto, 1, 267-273

Johnson, R.B. and de Graff, J. (1988). Principles of engineering geology. John Wiley and Sons

Kagoorlie Miner (1917). Sensational mining fatality, severe earth tremor causes fall of rock on Great Boulder mine, one man killed, others injured. Kalgoorlie, Western Australia

Kaiser, P.K., McCreath, D.R. and Tannant, D.D. (1996). Canadian rockburst support handbook. Geomechanics Research Centre, Laurentian University, Sudbury

Kramer, S.L. (1996). Geotechnical Earthquake Engineering. Prentice Hall

Mellick, A.G. (2007). Beaconsfield Investigation Report Prepared for the Coroner at the request of the Tasmanian Government concerning the incident resulting in the death of Larry Knight and the entrapment of Todd Rusell and Brant Webb which occurred at Beaconsfield Mine on 25th April 2006. Tasmanian Coroners Court, Australia

Mikula, P.A. and Poplawski, R.F. (1995). The seismic monitoring decision at Mt Charlotte gold mine. Underground Operators Conference, AusIMM, Kalgoorlie, 79-85

Obert, L. and Duval, W.I. (1967). Rock mechanics and the design of structures in rock. John Wiley and Sons
Ortlepp, W.D. (2006). RaSiM comes of age - a review of the contribution of the understanding and control of mine rockburst. Sixth International Symposium on Rockburst and Seismicity in Mines, Potvin and Hudyma eds., Perth, Australia, 9-11

Ortlepp, W.D. (1992). The design of support for the containment of rockburst damage in tunnels - an engineering approach. Rock Support in Mining and Underground Construction, Kaiser and McCreath eds., 593-609

Ortlepp, W.D. and Stacey, T.R. (1994).The need for yielding support in rockburst conditions, and realistic testing of rockbolts. International Workshop on Applied Rockburst Research, Cereceda and Van Sint Jan eds., Santiago, Chile, 249-259

Pizarro, N. (2014). Oficio N 5637 a Cámara de Diputados. Accidente fatal Señor José Orellana Ballesteros. CODELCO Chile

Potvin, Y. (2009). Strategies and tactics to control seismic risk in mines. Journal of the Southern African Institute of Mining and Metallurgy 109(3), 177-186

Potvin, Y., Wasseloo, J. and Heal, D. (2010). An interpretation of ground support capacity submitted to dynamic loading. Mining Technology 119(4), 233-245

Potvin, Y., Hadjigeorgiou, J. and Stacey, T.R. (2007). Introduction. Challenges in Deep and High Stress Mining, Potvin et al. eds., Australian Centre for Geomechanics, Perth, Australia, 1-12

Potvin, Y., Hudyma, M. and Jewell, R.J. (2000). Rockburst and seismic activity in underground Australian mines-an introduction to a new research project. ISRM International Symposium, International Society for Rock Mechanics, Melbourne, Australia Rahn, P.H. (1986). Engineering geology. An environmental approach. Elsevier

Rauert, N.S. and Tully, K.P. (1998). Integration of a microseismic monitoring system in mining the Pasminco Broken Hill Southern Cross Area. 7th AusIMM Undergroud Operators Conference, Townsville, Queensland, Australia

Walthan, T. (1999). Foundations of engineering geology. E \& FN Spon

Wyllie, D.C. (1999). Foundations on rock. $2^{\circ}$ ed. E \& FN Spon 